\title{
Stellar Core Collapse and Exotic Matter
}

\author{
Ken'ichiro Nakazato ${ }^{1}$ and Kohsuke Sumiyoshi ${ }^{2}$ \\ ${ }^{1}$ Department of Physics, Faculty of Science \& Technology, Tokyo University of Science, \\ Yamazaki 2641, Noda, Chiba 278-8510, Japan \\ email: nakazato@rs.tus.ac.jp \\ ${ }^{2}$ Numazu College of Technology, Ooka 3600, Numazu, Shizuoka 410-8501, Japan
}

\begin{abstract}
Some supernovae and gamma-ray bursts are thought to accompany a black hole formation. In the process of a black hole formation, a central core becomes hot and dense enough for hyperons and quarks to appear. In this study, we perform neutrino-radiation hydrodynamical simulations of a stellar core collapse and black hole formation taking into account such exotic components. In our computation, general relativity is fully considered under spherical symmetry. As a result, we find that the additional degrees of freedom soften the equation of state of matter and promote the black hole formation. Furthermore, their effects are detectable as a neutrino signal. We believe that the properties of hot and dense matter at extreme conditions are essential for the studies on the astrophysical black hole formation. This study will be hopefully a first step toward a physics of the central engine of gamma-ray bursts.
\end{abstract}

Keywords. black hole physics, dense matter, equation of state, hydrodynamics.

\section{Introduction}

The fate of stars with $M \gtrsim 25 M_{\odot}$ can be observationally split into two branches, namely, a hypernova branch and a faint-supernova branch, and they are both thought to form black holes eventually (Nomoto et al. 2006). The progenitors of the hypernova branch are thought to rotate strongly and associate with the gamma-ray bursts. On the other hand, nonrotating and weakly rotating massive stars are constituents of the faint-supernova branch and more massive stars are thought to result in so-called failed supernovae. In this study, we perform neutrino-radiation hydrodynamical simulations of a stellar core collapse and black hole formation taking into account hyperons and quarks. As a result, we find that the additional degrees of freedom soften the equation of state (EOS) of matter and promote the black hole formation. Further details of this study can also be found in our recent paper (Nakazato et al. 2012).

\section{Setups}

In the process of a black hole formation, a central core becomes hot and dense enough for hyperons and quarks to appear. In order to compute the stellar core collapse and black hole formation, we use the numerical code of general relativistic $\nu$-radiation hydrodynamics which solves the Boltzmann equation for neutrinos together with Lagrangian hydrodynamics under spherical symmetry. As an initial condition, the stellar model with $40 M_{\odot}$ and solar metallicity from the evolutionary calculation Woosley \& Weaver (1995) is adopted. To examine the effects of hyperons, we utilize the tables of EOS by Ishizuka et al. (2008), which are based on an $S U_{f}(3)$ extended relativistic mean field model and constructed as an extension of the EOS by Shen et al. (1998). Since it is undetermined whether the $\Sigma-N$ interaction is attractive or repulsive, we adopt both EOS sets with potential depths $\left(U_{\Lambda}, U_{\Sigma}, U_{\Xi}\right)=(-30 \mathrm{MeV},+30 \mathrm{MeV},-15 \mathrm{MeV})$ for the repulsive case 

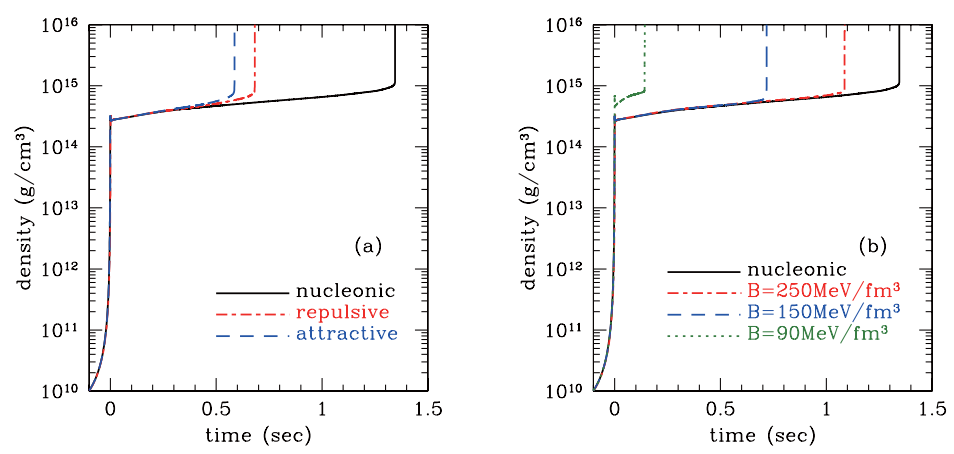

Figure 1. Time evolutions of the central baryon mass density for the collapse of models with (a) hyperons and (b) quarks. In both panels, the solid lines show the result for the model without hyperons and quarks.

and $(-30 \mathrm{MeV},-30 \mathrm{MeV},-15 \mathrm{MeV})$ for the attractive case. As for the quark effects, we investigate the MIT bag model with the EOS by Shen et al. (1998) as in Nakazato et al. (2008). In this model, the ambiguities of the interaction are encapsulated in one parameter called bag constant, $B$, and we examine $B=90,150$ and $250 \mathrm{MeV} / \mathrm{fm}^{3}$. Note that, for the EOS's with hyperons or quarks, the maximum masses of neutron stars are smaller than $1.97 \pm 0.04 M_{\odot}$, the mass of the binary millisecond pulsar J1614-2230 Demorest et al. (2010). While our focus is on investigating systematic differences due to the interactions of hyperons and quarks, further studies on the EOS are also important.

\section{Results}

In Figure 1, we show the time profiles of the central baryon mass density. The core is bounced once and then recollapses to a black hole. We can recognize that the time interval between the bounce and black hole formation gets shorter as we put additional degrees of freedom, hyperons or quarks. As for the hyperonic models, the black hole is formed earlier for the attractive case because $\Sigma$ hyperons appear more easily and soften the EOS. On the other hand, for the quark models, the time interval of the lower bag constant case is shorter because the phase transition occurs at lower density and triggers the black hole formation. Note that the density at the bounce of the model with $B=90 \mathrm{MeV} / \mathrm{fm}^{3}$ differs from those of other models because the transition occurs already at the bounce. Since the duration of neutrino emission corresponds to the time interval between the bounce and black hole formation, we may be able to probe observationally the effects of hyperons and quarks using the difference of the time interval in future.

\section{References}

Demorest, P. B., Pennucci, T., Ransom, S. M., Roberts, M. S. E., \& Hesseles, J. W. T. 2010, Nature, 467, 1081

Ishizuka, C., Ohnishi, A., Tsubakihara, K., Sumiyoshi, K., \& Yamada, S. 2008, J. of Phys. G, 35,085201

Nakazato, K., Furusawa, S., Sumiyoshi, K., Ohnishi, A., Yamada, S., \& Suzuki, H. 2012, ApJ, 745,197

Nakazato, K., Sumiyoshi, K., \& Yamada, S. 2008, Phys. Rev. D, 77, 103006

Nomoto, K., Tominaga, N., Umeda, H., Kobayashi, C., \& Maeda, K. 2006, Nucl. Phys., A, 777, 424

Shen, H., Toki, H., Oyamatsu, K., \& Sumiyoshi, K. 1998, Prog. Theor. Phys., 100, 1013

Woosley, S. E. \& Weaver, T. A. 1995, ApJS, 101, 181 